\title{
Malignant Vaginal Mixed Epithelial and Mesenchymal Neoplasm
}

National Cancer Institute

\section{Source}

National Cancer Institute. Malignant Vaginal Mixed Epithelial and Mesenchymal

Neoplasm. NCI Thesaurus. Code C40276.

A malignant neoplasm that arises from the vagina and is characterized by the presence of an epithelial and a mesenchymal component. This category includes adenosarcoma, carcinosarcoma, and malignant mixed tumor resembling synovial sarcoma. 